ISSN 1112-9867

Available online at http://www.jfas.info

\title{
ANALYSIS OF THE PHENOTYPIC VARIABILITY OF SOME VARIETIES OF DURUM WHEAT (TRITICUM DURUM DESF) TO IMPROVE THE EFFICIENCY OF PERFORMANCE UNDER THE CONSTRAINING CONDITIONS OF SEMI- ARID ENVIRONMENTS
}

\author{
L. Haddad ${ }^{1, *}$, H. Bouzerzour ${ }^{2}$, A. Benmahammed ${ }^{2}$, H. Zerargui ${ }^{3}$, A. Hannachi ${ }^{4}$, A. \\ Bachir $^{3}$, M. Salmi ${ }^{1}$, A. Oulmi ${ }^{2}$, H. Nouar ${ }^{2}$, Z. Laala ${ }^{1}$ \\ ${ }^{1}$ Agronomy Department, Faculty of Life and Natural Sciences, LVRBN Laboratory, \\ Ferhat Abbas University, Setif 1, Algeria \\ ${ }^{2}$ Ecology and Biology Department, Faculty of Life and Natural Sciences, LVRBN \\ Laboratory, Ferhat Abbas University, Setif 1, Algeria \\ ${ }^{3}$ Field Crop Institute Agricultural Research Station (ITGC), Setif, Algeria. \\ ${ }^{4}$ Algerian National Institute of Agronomic Research (INRAA), Research Unit of Setif, \\ Algeria
}

Received: 08 June 2016 / Accepted: 31 August 2016 / Published online: 01 September 2016

\begin{abstract}
The experiment was conducted during three growing seasons and two planting dates. The cultivation site is placed at the ITGC Setif characterized by a semi-arid environment. The objective of the study is the analysis of phenotypic variability of traits measured for 15 varieties of durum wheat, through the average effects, to decline the ways, characters and varieties could play in favour of performance under the constraining semi-arid conditions. The year effect indicates that given the difficulty of predicting the performance enabled by years, it then makes sense to go straight for this performance within genotypes. Analysis of the effect genotype highlights characters connected to performance and is the Setifis variety that lends itself well. For the effect of sowing date, it is that early sowing promotes a better expression of the characteristics compared to late sowing.
\end{abstract}

Keywords: Effect; genotype; year; date; variation; characters.

Author Correspondence, e-mail: Haddadleila2016@gmail.com

doi: http://dx.doi.org/10.4314/jfas.v8i3.19 


\section{INTRODUCTION}

Le blé dur (Triticum turgidum L. var. durum) est l'une des principales ressources alimentaires de l'humanité. Il est la première céréale cultivée en Algérie, en termes de superficies et de production, occupant la moitié d'une superficie de 3 millions hectares, destinés à la céréaliculture. La production qui avoisine les 1.6 millions de tonnes ne couvre que 30 à 35\% des besoins. La cause principale de la faiblesse de la production est le bas niveau des rendements atteint actuellement et qui, quoiqu'en augmentation depuis 1990, reste en deçà de ce qu'il devrait être, ne dépassant pas une moyenne de 1.5 t/ha [1]. La majeure partie des emblavures du blé dur est concentrée sur les hautes plaines, région caractérisée par de l'altitude (700 à $1100 \mathrm{~m}$ ), des hivers froids, un régime pluviométrique faible et irrégulier, associés à l'apparition de vents chauds et desséchants en fin de cycle de la culture [2]. Les changements climatiques risquent d'exagérer l'effet de ces contraintes qui s'ajoutent aux contraintes d'ordre techniques qui relèvent de l'application d'itinéraires techniques souvent peu ambitieux et de l'utilisation de variétés non adaptées [3]. En effet les nouvelles variétés adoptées sont sélectionnées sur la base de leur potentiel de rendement sans tenir compte des caractères adaptatifs qui sont des régulateurs de la production en milieux variables [4]. De plus, dans ces milieux, La variation des performances génotypiques, engendrée par l'interaction génotype $\mathrm{x}$ milieux, rend difficile la sélection et la recommandation des génotypes performants $[5,6]$. Les alternatives qui s'offrent, pour contrer ces contraintes, sont peu nombreuses et difficiles à mettre en œuvre. Il se trouve que la stratégie la plus favorable est de développer des variétés dont le rendement est élevé et qui soient tolérantes aux diverses contraintes qui caractérisent les milieux de production $[7,6]$. Pour ce faire, il devient plus que nécessaire d'avoir des connaissances plus approfondies sur de multiples et d'éventuels géniteurs de blé dur sous conditions limitantes pour développer de nouvelles variétés capables de résister au manque d'eau associé aux températures basses et élevées et à la salinité [8]. Les futures variétés doivent avoir, en plus de bonnes valeurs agronomiques et technologiques, la capacité de valoriser, au mieux, les spécificités des différents terroirs et itinéraires techniques. Elles doivent réagir positivement avec la diversité des situations dans lesquelles elles sont placées où dans lesquelles la variation de milieu les place $[9,10,11,6,12]$. Cette étude a pour objectif d'analyser la variabilité phénotypique de quelques variétés de blé dur (Triticum durum Desf.), à travers les effets moyens années, génotypes et dates de semis, afin de déterminer les méthodes, caractères et variétés susceptibles de jouer en faveur du rendement sous les conditions contraignantes de l'environnement semi-arides. 


\section{EQUIPEMENTS ET METHODES}

\subsection{Site, Matériel végétal et dispositif expérimental}

L'étude de la variabilité s'est portée sur 15 variétés de blé dur (Tableau 1), elle a été menée sur trois campagnes agricoles $(2012 / 2013,2013 / 2014$ et 2014/2015) avec deux dates de semis par campagnes au niveau de la station expérimentale agricole de l'ITGC de Sétif (SEA-ITGC Sétif).

Tableau 1. Liste des génotypes de blé dur évalués au cours des campagnes 2012/13, 2013/14 et 2014/15 sur le site expérimental de la station ITGC de Sétif.

\begin{tabular}{|l|l|l|l|}
\hline Nom & Pedigree & Croisement & Sélection \\
\hline Massara & Mrb $_{3} / 4 / \mathrm{Bye} * 2 / \mathrm{Tc} / 2 / \mathrm{Zb} / \mathrm{W} / 3 / \mathrm{Cit}$ & Cimmyt-Icarda & ITGC \\
\hline Cyprus 2 & Gdovz $_{51} / \mathrm{Cit} / / \mathrm{Ruff} / \mathrm{Fg} / 3 / \mathrm{Ggovz}_{449}$ & Cimmyt-Icarda & ITGC \\
\hline MBB & Variété locale & Land race Alg & ITGC \\
\hline Waha & Plc/Ruff//Gta's/3/Rolette CM 17904 & Cimmyt-Icarda & ITGC \\
\hline Gta dur & Crane/4/PolonicumPI ${ }_{185309} / /$ T.glutin en/2* Tc60/3/Gll & Cimmyt-Icarda & ITGC \\
\hline Vitron & Turkey77/3/Jori/ Anhinga//Flamingo & Cimmyt-Icarda & ITGC \\
\hline Bousselam & Heider/Martes//Huevos de Oro & Cimmyt-Icarda & ITGC \\
\hline Massinissa & Ofanto/Bousselam & SEA-ITGC-Sét & ITGC \\
\hline Megress & Ofanto/Waha//MBB & SEA-ITGC-Sét & ITGC \\
\hline Setifis & Bousselam/Ofanto & SEA-ITGC-Sét & ITGC \\
\hline Mansoura & Chinese spring/MBB & SEA-ITGC-Sét & ITGC \\
\hline Tajdid & Ofanto/Bousselam's & SEA-ITGC-Sét & ITGC \\
\hline Moustakbel & Gta dur/Ofanto & SEA-ITGC-Sét & ITGC \\
\hline Boutaleb & Hedba/Ofanto & SEA-ITGC-Sét & ITGC \\
\hline
\end{tabular}

La station expérimentale appartient à l'étage bioclimatique semi-aride caractérisé par un climat continental typiquement méditerranéen. Le sol du site expérimental est de type brun calcaire, de la classe des sols bruns steppiques, qui se caractérise par un $\mathrm{pH}$ de 8.2 et un taux de matière organique de $1.35 \%$. Il a une composition de $13.9 \%$ de sable, $41.3 \%$ de limon et $44.7 \%$ d'argile [13].

Le matériel végétal a été semé dans un dispositif en blocs complètement randomisés avec trois répétitions, sur des parcelles élémentaires de $6 \mathrm{~m}^{2}$. La première date de semis est réalisée au mois de décembre et la seconde au mois de janvier (ce qui implique que la première date de semis est considérée comme précoce par comparaison à la seconde considérée alors comme tardive). La densité de semis est de 300 graines au $\mathrm{m}^{2}$. Les essais dont le précédent cultural est une légumineuse pour la première campagne et une jachère pour la seconde et la troisième campagne, sont fertilisés avec une fumure de fond, le MAP à 52\% de phosphore, à 
raison de $80 \mathrm{Kg} / \mathrm{ha}$ avant le semis. Comme fumure de couverture, l'urée (35\%), a été épandu au stade tallage à raison de $100 \mathrm{~kg} / \mathrm{ha}$. Le contrôle des adventices est réalisé chimiquement avec l'herbicide Brumby $80 \mathrm{EC}(80 \mathrm{~g} / \mathrm{l}$ Clodinafop propargyl $+20 \mathrm{~g} / \mathrm{l}$ Cloquintocel mexyl), appliqué au stade 2-3 feuilles contre les graminées adventices à raison de 0,75 1/ha et le Granstar 75 DF (Methyl Tribenuron) contre les adventices dicotylédones à raison de 12,5 g/ha.

\subsection{Mesures et notations}

Les mesures et notations suivantes ont été réalisées par parcelle élémentaire.

\subsubsection{Nombre de plants}

Le nombre de plants installés a été compté à la levée sur 3 stations de $1 \mathrm{~m}$ linéaire de long en diagonale de la parcelle élementaire.

\subsubsection{Nombre de talles herbacées}

Le nombre de talles émises par plante a été déterminé au stade tallage, sur les mêmes stations qui ont servi à la détermination du nombre de plantes par unité de surface.

\subsubsection{Hauteur de la végétation}

La hauteur est prise du sol au sommet de l'épi, barbes non incluses, avec une ruban mètre.

\subsubsection{Surface foliaire}

La surface foliaire de la feuille étendard est déterminée par produit de la longueur par la plus grande largeur de la feuille étendard, mesurées sur un échantillon de 10 feuilles prises au hasard au stade épiaison par le coefficient de 0.704. Ce coefficient est rapporté par Spagnoletti-Zeuli et Qualset (1990), il représente le coefficient de régression de la surface foliaire déduite par le produit L x 1 sur celle déduite par la méthode de la photocopie sur papier grammage:

$$
\text { SF }\left(\mathbf{c m}^{2}\right)=0.704 \times(L \times l)
$$

Où $\mathrm{SF}=$ surface moyenne de la feuille étendard $\left(\mathrm{cm}^{2}\right), \mathrm{L}=$ longueur moyenne de la feuille étendard $(\mathrm{cm}), l=$ largeur moyenne de la feuille étendard $(\mathrm{cm})$.

\subsubsection{Longueur du col de l'épi}

La longueur du col est estimée par la longueur qui sépare le deux noued point d'attache de la feuille étendard et les base de l'épi, cm, à maturité.

\subsubsection{Durée de la phase végétative}

La durée de la phase végétative est estimée en nombre de jours calendaires comptés à partir du 1èr janvier à la date de sortie de $50 \%$ des épis par unités de surface ou par parcelle élémentaire.

\subsubsection{Biomasse aérienne}


La biomasse aérienne est déterminée au stade maturité à partir du poids des botillions provenant de la récolte d'un ségment de rang de $1 \mathrm{~m}$ de long par parcelle élémentaire. Elle est exprimée en $\mathrm{g} / \mathrm{m}^{2}$, sans passage à l'étuve.

\subsubsection{Nombre et poids des épis}

Le nombre et le poids des épis produits par unité de surface sont déduits du comptage et pesage du nombre d'épis présent dans les bottillons de végétation récoltés à maturité. Ils sont exprimés en nombre d'épis $/ \mathrm{m}^{2}$ en $\mathrm{g} / \mathrm{m}^{2}$.

\subsubsection{Rendement grain et composantes du rendement}

Le rendement grain est déterminé par le poids des graines issues du battage des épis comptés par bottillon de végétation. Il est exprimé en $\mathrm{g} / \mathrm{m}^{2}$. 250 graines sont comptées à partir du produit du battage des épis et pesées pour déterminer la composante du rendement qui est le poids de 1000 grains. Le nombre de grains/ épi (NGE) et celui de graines produites par $\mathrm{m}^{2}$ $\left(\mathrm{NGM}^{2}\right)$ sont estimés par calcul direct à partir des estimations du rendement grain (RDT, $\mathrm{g} / \mathrm{m}^{2}$ ), du poids de 1000 grains (PMG, g/1000 grains) et du nombre d'épis $/ \mathrm{m}^{2}\left(\mathrm{NE}, \mathrm{nbre} / \mathrm{m}^{2}\right)$ :

$$
\begin{gathered}
\text { NGE }=(1000 \text { RDT }) /(\text { PMG } \times \text { NE }) ~(2) \\
\text { et }
\end{gathered}
$$$$
\mathrm{NGM}^{2}=\text { NGE } X \text { NE (3) }
$$

\subsection{Analyse des données}

Les données collectées sont soumises à une simple analyse de la variance (Anova) pour déterminer les effets moyens années, génotypes, dates de semis ainsi que les interactions qui s'y engendrent. Le logiciel utilisé est le CropStat 7.2.3 [14].

\section{RESULTATS ET DISCUSSION}

\subsection{Variabilité phénotypique des caractères mesurés}

L'analyse de la variance des caractères mesurés indique des effets moyens et des interactions significatives pour l'ensemble des caractères analysés (Tableau 2). 
Tableau 2. Valeurs du test F de l'analyse de la variance des caractères mesurés

\begin{tabular}{|c|c|c|c|c|c|c|c|c|c|c|}
\hline Source & Traitement & Rép & $\mathrm{A}$ & $\mathrm{D}$ & $A \times D$ & $\mathrm{G}$ & $A x G$ & DxG & AxDxG & Erreur \\
\hline dll & 89 & 2 & 2 & 1 & 2 & 14 & 28 & 14 & 28 & 178 \\
\hline PLT & $* *$ & $\mathrm{~ns}$ & $* *$ & $* *$ & $* *$ & $* *$ & $* *$ & $* *$ & $* *$ & 218.2 \\
\hline $\mathrm{TH}$ & $* *$ & $\mathrm{~ns}$ & $* *$ & $* *$ & $* *$ & $* *$ & $* *$ & $* *$ & $* *$ & 1558.5 \\
\hline HT & $* *$ & ns & $* *$ & $* *$ & $* *$ & $* *$ & $* *$ & $* *$ & $* *$ & 2.9 \\
\hline SF & $* *$ & ns & $* *$ & $* *$ & $* *$ & $* *$ & $* *$ & $* *$ & $* *$ & 0.6 \\
\hline LC & $* *$ & ns & $* *$ & $* *$ & $* *$ & $* *$ & $* *$ & $* *$ & $* *$ & 0.7 \\
\hline PREC & $* *$ & ns & $* *$ & $* *$ & $* *$ & $* *$ & $* *$ & $* *$ & $* *$ & 0.1 \\
\hline $\mathrm{BIO}$ & $* *$ & ns & $* *$ & $* *$ & $* *$ & $* *$ & $* *$ & $* *$ & $* *$ & 2484.5 \\
\hline $\mathrm{PE}$ & $* *$ & ns & $* *$ & $* *$ & $* *$ & $* *$ & $* *$ & ** & $* *$ & 324.8 \\
\hline $\mathrm{NE}$ & $* *$ & ns & $* *$ & $* *$ & $* *$ & $* *$ & $* *$ & $* *$ & $* *$ & 398.5 \\
\hline NGE & $* *$ & ns & $* *$ & $* *$ & $* *$ & $* *$ & $* *$ & $* *$ & $* *$ & 3.2 \\
\hline NGM & $* *$ & ns & $* *$ & $* *$ & $* *$ & $* *$ & $* *$ & $* *$ & $* *$ & 201816 \\
\hline PMG & $* *$ & $*$ & $* *$ & $* *$ & $* *$ & $* *$ & $* *$ & $* *$ & $* *$ & 27.3 \\
\hline RDT & $* *$ & $\mathrm{~ns}$ & $* *$ & $* *$ & $* *$ & $* *$ & $* *$ & $* *$ & $* *$ & 36119 \\
\hline
\end{tabular}

$\mathrm{ns}, *=$ effets non significatif et significatif au seuil de $5 \%$, respectivement.

\subsubsection{Effet année}

L'analyse de l'effet moyen année montre que les campagnes 2014 et 2015 diffèrent fortement pour l'expression de l'ensemble des caractères (Tableau 3). La réduction des valeurs moyennes des variables mesurées varie de $20 \%$ à $80 \%$ des valeurs maximales observées au cours de la campagne 2013 (Figure 1).

Les réductions les plus importantes observées sont celles du rendement en grains, de la biomasse aérienne, du nombre de grains par $\mathrm{m}^{2}$, du poids des épis, du nombre de grains par épi, de la longueur du col, de la surface de la feuille étendard et de la hauteur de la végétation (Tableau 3, Figure 1). Ces résultats indiquent que ce n'est pas uniquement le rendement en grains qui est variable en fonction des campagnes mais également l'ensemble des caractères qui constituent l'architecture de la plante (Tableau 3).

Tableau 3. Valeurs moyennes des variables mesurées de l'effet année

\begin{tabular}{|l|l|l|l|l|l|l|l|l|l|l|l|l|l|}
\hline Année & PLT & Tll & PHT & SF & LC & DPV & BIO & PE & NE & NGE & NGM $^{2}$ & PMG & RDT \\
\hline 2013 & 223.4 & 807.3 & 82.9 & 21.6 & 31.8 & 124.3 & 1180.8 & 710.4 & 389.1 & 30.7 & 11771.5 & 41.9 & 490.4 \\
\hline 2014 & 236.7 & 788.1 & 49.7 & 13.4 & 15.5 & 120.1 & 350.5 & 125.7 & 210.4 & 10.4 & 2181.0 & 35.7 & 77.9 \\
\hline 2015 & 237.3 & 569.2 & 43.4 & 10.9 & 14.6 & 126.1 & 515.2 & 296.2 & 311.1 & 15.9 & 4873.4 & 39.9 & 192.8 \\
\hline Ppds 5\% & 4.3 & 11.6 & 0.5 & 0.2 & 0.2 & 0.00 & 14.6 & 5.3 & 5.8 & 0.5 & 120.6 & 0.5 & 4.1 \\
\hline
\end{tabular}

$\mathrm{PLT}=$ Plantes levées $/ \mathrm{m}^{2}, \mathrm{Tll}=$ talles herbacées $/ \mathrm{m}^{2}, \mathrm{PHT}=$ hauteur de la plante, $\mathrm{cm} ; \mathrm{SF}=$ surface de la feuille étendard, $\mathrm{cm}^{2}$; $\mathrm{LC}=$ longueur du col de l'épi, $\mathrm{cm}$; DPV=durée de la phase végétative, jours ; $\mathrm{BIO}=$ biomasse aéreinene mesurée à maturité, $\mathrm{g} / \mathrm{m}^{2} ; \mathrm{PE}=$ Poids de épis $/ \mathrm{m}^{2}, \mathrm{~g} / \mathrm{m}^{2} ; \mathrm{NE}=$ Nombre d'épis par $\mathrm{m}^{2}, \mathrm{NGM}^{2}=$ Nombre de grains par $\mathrm{m}^{2}, \mathrm{PMG}=$ Poids de 1000 rains, $\mathrm{g} ; \mathrm{RDT}=$ Rendement en grains, $\mathrm{g} / \mathrm{m}^{2}$.

Ainsi le rendement en grains chute de 490.4 à $77.9 \mathrm{~g} / \mathrm{m}^{2}$, soit une réduction de $84.1 \%$ en 2014 et de $60.7 \%$ en 2015. Le nombre de grains par $\mathrm{m}^{2}$ baisse de 11771.5 à 2181.0, soit une réduction de $81.5 \%$ en 2014 et de $58.6 \%$ en 2015 . La biomasse chute de 1180.8 à $350.5 \mathrm{~g} / \mathrm{m}^{2}$, soit une réduction de $70.2 \%$ en 2014 et de $56.4 \%$ en 2015 ( Tableau 3, Figure 1). 
Par contre le nombre de plantes levées montre une variation de l'ordre de $6.0 \%$, alors que le nombre de talles varie de 807.3 à 569.2 talles par $\mathrm{m}^{2}$ entre 2013 et 2015. Ces valeurs moyennes restent largement supérieurs aux valeurs moyennes des talles montantes en épis par $\mathrm{m}^{2}$ qui varient de 389.1 à 210.4 épis $/ \mathrm{m}^{2}$, soit des réductions de $45.9 \%$ en 2014 et $20.0 \%$ en 2015 ( Tableau 3, Figure 1). Du point de vu sélection, il est difficile de prédire les performances permises par une année donnée, de ce fait, on ignore cette variation d'origine environnementale, tout en cherchant le génotype qui, tout en étant performant, soit le moins sensible possible à cette variation.

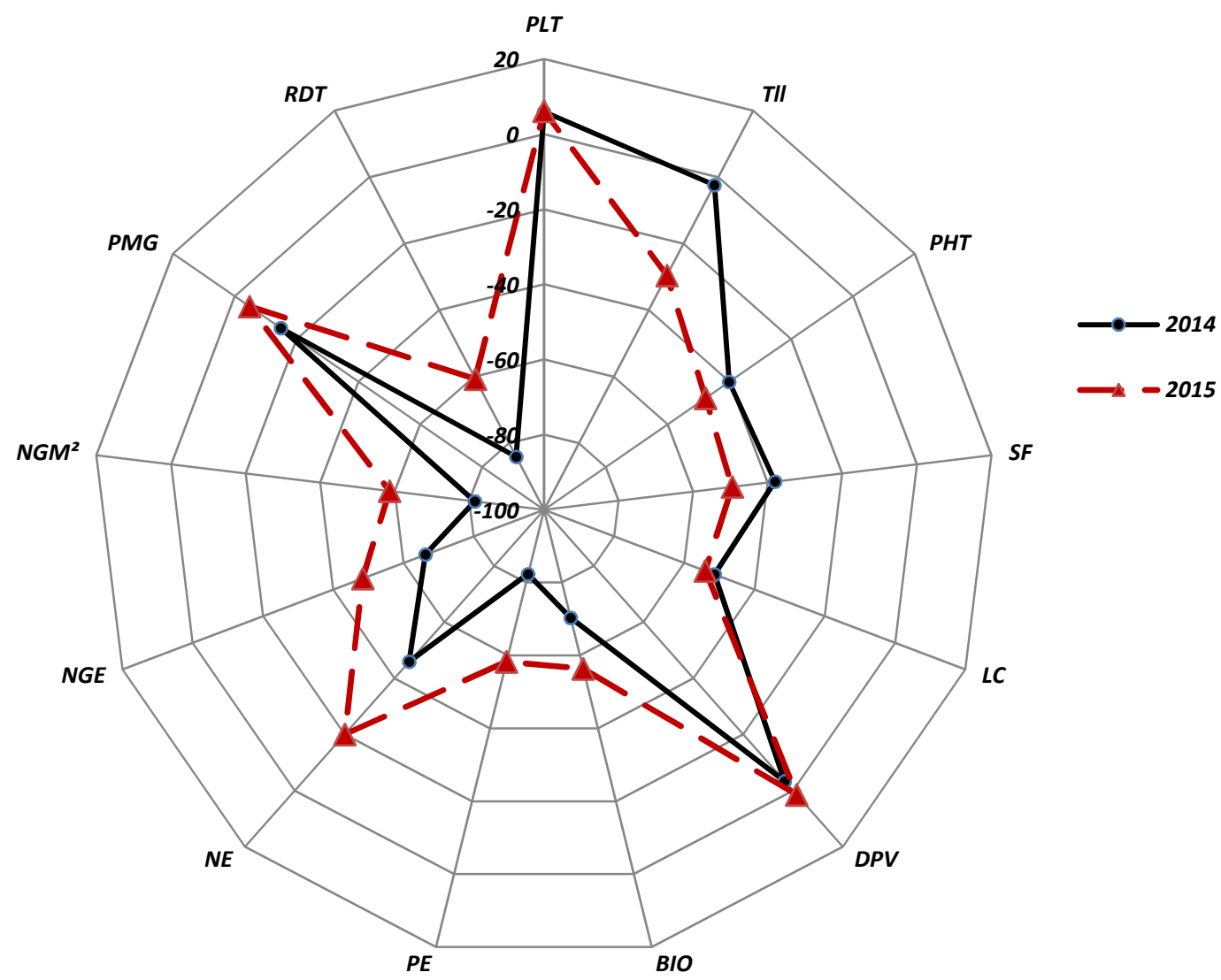

Fig.1. Réduction des valeurs moyennes des variables mesurées des campagnes 2014 et 2015 comparativement à celles de 2013. (PLT= Plantes levées $/ \mathrm{m}^{2}, \mathrm{Tll}=$ talles herbacées $/ \mathrm{m}^{2}, \mathrm{PHT}=$ hauteur de la plante, $\mathrm{cm} ; \mathrm{SF}=$ surface de la feuille étendard, $\mathrm{cm}^{2}$; LC= longueur du col de l'épi, $\mathrm{cm}$; DPV=durée de la phase végétative, jours ; $\mathrm{BIO}=$ biomasse aéreinene mesurée à maturité, $\mathrm{g} / \mathrm{m}^{2} ; \mathrm{PE}=$ Poids de épis $/ \mathrm{m}^{2}, \mathrm{~g} / \mathrm{m}^{2} ; \mathrm{NE}=$ Nombre d'épis par $\mathrm{m}^{2}, \mathrm{NGM}^{2}=$ Nombre de grains par $\mathrm{m}^{2}, \mathrm{PMG}=$ Poids de 1000 rains, $\mathrm{g} ; \mathrm{RDT}=$ Rendement en grains, $\mathrm{g} / \mathrm{m}^{2}$ )

\subsubsection{Effet génotype}

Les moyennes des variables mesurées, calculées sur l'ensemble des dates et années, montrent une importante variabilité. Ainsi la moyenne du nombre de plants levés est de 232.5 avec un 
maxima de 264.5 et un minima de 206.0 plants $/ \mathrm{m}^{2}$. Le nombre de talles herbacées varie de 851.3 à 624.3 , avec une moyenne de 721.5 talles $/ \mathrm{m}^{2}$. La hauteur de la végétation varie de 76.1 à 51.6, avec une moyenne de $58.9 \mathrm{~cm}$. La surface de la feuille étendard varie de 19.5 à 12.5 , avec une moyenne de $15.3 \mathrm{~cm}^{2}$. La longueur du col de l'épi varie de 25.5 à 18.8 , avec une moyenne de $20.6 \mathrm{~cm}$ (Tableau 4).

La durée de la phase végétative varie de 131.3 à 119.2, avec une moyenne de 123.5 jours. La biomasse accumulée à maturité varie de 955.1 à 576.3, avec une moyenne de $682.2 \mathrm{~g} / \mathrm{m}^{2}$. Le poids des épis varie de 450.6 à 306.1 , avec une moyenne de $377.4 / \mathrm{m}^{2}$. Le nombre d'épis varie de 335.0 à 265.5, avec une moyenne de 303.5 épis $/ \mathrm{m}^{2}$. Le nombre de grains par épi varie de 23.1 à 14.2, avec une moyenne de 19.0 grains/épi (Tableau 4).

Le nombre de grains par $\mathrm{m}^{2}$ varie de 7856.1 à 5255.8 , avec une moyenne de 6275.3 grains $/ \mathrm{m}^{2}$. Le poids de 1000 grains varie de 44.6 à 35.4, avec une moyenne de 39.2 g, et le rendement en grains varie de 304.2 à 202.8 avec une moyenne de 253.7 gm² $^{2}$ (Tableau 4). L'analyse de l'amplitude, qui est la différence entre les valeurs moyennes maximales et les valeurs moyennes minimales, exprimée en $\%$ de la moyenne de chaque caractère, montre les possibilités de sélection au sein de l'échantillon de variétés étudiées (Figure 2).

Tableau 4. Valeurs moyennes des variables mesurées dues à l'effet génotype

\begin{tabular}{|l|l|l|l|l|l|l|l|l|l|l|l|l|l|}
\hline Geno & PLT & Tll & PHT & SF & LC & DPV & BIO & PE & NE & NGE & NGM $^{2}$ & PMG & RDT \\
\hline MAS & 235.7 & 851.3 & 60.1 & 14.3 & 23.2 & 119.2 & 645.8 & 418.2 & 328.9 & 22.0 & 7522.7 & 37.0 & 292.4 \\
\hline Sét13 & 206.0 & 624.8 & 76.1 & 12.5 & 25.5 & 131.3 & 955.1 & 430.5 & 299.8 & 22.3 & 6943.1 & 38.0 & 274.1 \\
\hline CYP2 & 217.9 & 657.5 & 54.6 & 19.5 & 20.1 & 122.5 & 576.3 & 306.1 & 282.1 & 19.8 & 5542.8 & 37.9 & 217.1 \\
\hline MBB & 255.9 & 727.8 & 75.2 & 15.5 & 22.9 & 129.5 & 762.3 & 315.9 & 326.1 & 16.5 & 5681.8 & 35.7 & 214.0 \\
\hline WAH & 219.4 & 624.3 & 56.3 & 14.7 & 20.4 & 122.0 & 595.5 & 349.6 & 281.4 & 20.6 & 6438.8 & 36.9 & 238.1 \\
\hline GTA & 220.0 & 785.0 & 55.1 & 18.1 & 19.9 & 122.3 & 697.3 & 370.5 & 315.6 & 18.7 & 6508.0 & 37.9 & 256.1 \\
\hline VIT & 208.4 & 749.2 & 54.5 & 15.9 & 19.6 & 120.7 & 661.6 & 396.5 & 301.7 & 20.6 & 6788.2 & 37.4 & 266.3 \\
\hline MEG & 264.5 & 733.5 & 54.0 & 15.3 & 19.6 & 120.3 & 704.0 & 380.5 & 329.6 & 16.7 & 5943.0 & 42.9 & 253.4 \\
\hline SET & 255.6 & 797.8 & 55.7 & 13.0 & 19.4 & 123.5 & 763.5 & 450.6 & 309.3 & 23.1 & 7856.1 & 37.9 & 304.2 \\
\hline BOU & 221.1 & 737.5 & 56.3 & 14.5 & 18.8 & 124.3 & 707.4 & 412.7 & 310.9 & 18.1 & 6799.4 & 42.5 & 292.4 \\
\hline MAN & 257.9 & 790.6 & 55.1 & 13.0 & 21.3 & 124.3 & 626.9 & 360.2 & 335.0 & 14.2 & 5849.0 & 39.0 & 229.5 \\
\hline MAI & 238.1 & 648.1 & 57.5 & 17.0 & 20.0 & 120.5 & 611.9 & 364.5 & 265.5 & 21.1 & 5905.0 & 41.3 & 253.2 \\
\hline TJD & 238.5 & 677.4 & 51.5 & 14.4 & 20.0 & 126.3 & 604.1 & 326.4 & 276.6 & 17.3 & 5255.8 & 35.4 & 202.8 \\
\hline MOU & 235.6 & 682.2 & 55.2 & 16.5 & 19.5 & 121.5 & 638.0 & 355.6 & 288.4 & 16.6 & 5354.7 & 43.3 & 244.5 \\
\hline BTA & 212.5 & 736.1 & 62.6 & 15.2 & 19.3 & 123.8 & 683.2 & 423.6 & 302.1 & 17.5 & 5740.8 & 44.6 & 267.8 \\
\hline MG & 232.5 & 721.5 & 58.6 & 15.3 & 20.6 & 123.5 & 682.2 & 377.4 & 303.5 & 19.0 & 6275.3 & 39.2 & 253.7 \\
\hline Maxi & 264.5 & 851.3 & 76.1 & 19.5 & 25.5 & 131.3 & 955.1 & 450.6 & 335.0 & 23.1 & 7856.1 & 44.6 & 304.2 \\
\hline mini & 206.0 & 624.3 & 51.5 & 12.5 & 18.8 & 119.2 & 576.3 & 306.1 & 265.5 & 14.2 & 5255.8 & 35.4 & 202.8 \\
\hline Range & 58.6 & 226.9 & 24.6 & 7.0 & 6.8 & 12.2 & 378.8 & 144.6 & 69.5 & 8.9 & 2600.4 & 9.1 & 101.4 \\
\hline Ppds5\% & 9.7 & 25.9 & 1.1 & 0.5 & 0.5 & 0.0 & 32.6 & 11.8 & 13.1 & 1.2 & 269.7 & 1.1 & 9.2 \\
\hline
\end{tabular}

Mas= Massara, Set13= Sétif2013, Cyp2= Cyprus2, MBB= Mohammed Ben Bachir, WAH= Waha, GTA= Gaviota durum, $\mathrm{VIT}=$ Vitron, $\mathrm{MEG}=$ Megress, $\mathrm{SET}=$ Setifis, $\mathrm{BOU}=$ Bousselam, MAN= Mansoura, $\mathrm{MAI}=$ Massinissa, $\mathrm{TJD}=\mathrm{Tajdid}, \mathrm{MOU}=$ Moustakbel, BTA= Boutaleb

Ainsi, en moyenne de l'ensemble des années et dates de semis évaluées, il est possible de réduire de la durée de la phase végétative de $10 \%$ de la moyenne générale de cette variable, 
comme il est aussi possible d'améliorer la biomasse aérienne de $56 \%$ et le rendement en grains de $40 \%$ de la moyenne générale de cette variable (Figure 2, Tableau 4).

Ces différences entre génotypes représentent la moyenne des différences de réponses aux années et dates de semis, qui ne sont pas toujours constantes suite à la présence de l'interaction des génotypes avec les dates et les années, comme il est indiqué par l'analyse de la variance des différents caractères (Tableau 2).

Cependant cet effet moyen génotype indique que les variétés les plus hautes sont Sétif 2013 et MBB. Cyprus 2 présente la feuille étendard aux larges dimensions. Sétif ${ }_{2013}$, en plus de sa haute taille, présente aussi les valeurs les plus élevées pour la longueur du col, la durée de la phase végétative et la biomasse aérienne mesurée à maturité. Par contre Setifis présente les valeurs, les plus élevées, pour le poids des épis, les nombres de grains par épi et par $\mathrm{m}^{2}$ et le rendement en grains (Tableau 4).

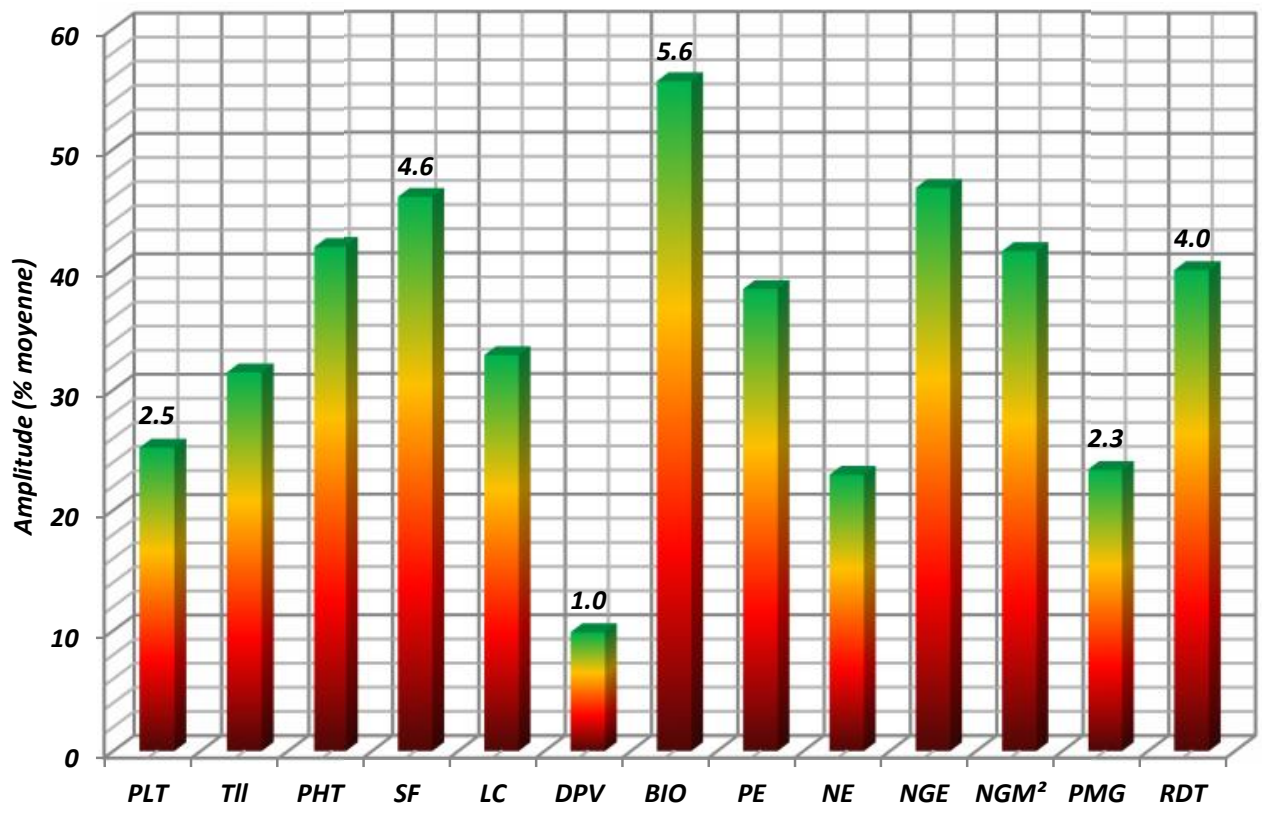

Fig.2. Amplitude exprimée en \% de la moyenne de l'ensemble des variétés des variables mesurées. $\left(\mathrm{PLT}=\mathrm{Plantes}\right.$ levées $/ \mathrm{m}^{2}, \mathrm{Tll}=$ talles herbacées $/ \mathrm{m}^{2}, \mathrm{PHT}=$ hauteur de la plante, $\mathrm{cm} ; \mathrm{SF}=$ surface de la feuille étendard, $\mathrm{cm}^{2} ; \mathrm{LC}=$ longueur du col de l'épi, $\mathrm{cm}$; DPV=durée de la phase végétative, jours ; BIO=biomasse aéreinene mesurée à maturité, $\mathrm{g} / \mathrm{m}^{2} ; \mathrm{PE}=$ Poids de épis $/ \mathrm{m}^{2}, \mathrm{~g} / \mathrm{m}^{2} ; \mathrm{NE}=$ Nombre d'épis par $\mathrm{m}^{2}, \mathrm{NGM}^{2}=$ Nombre de grains $\mathrm{par}^{2}$, PMG= Poids de 1000 rains, $\mathrm{g} ; \mathrm{RDT}=$ Rendement en grains, $\mathrm{g} / \mathrm{m}^{2}$ ).

En moyenne des dates et des années, les génotypes évalués se classent en deux groupes qui sont constitués des génotypes Massara, Waha, Setifis, Gaviota durum, Sétif ${ }_{2013}$, Vitron et Bousselam pour le groupe 1 et Cyprus $_{2}$, MBB, Megress, Mansoura, Massinissa, Tajdid, Moustakbel et Boutaleb pour le groupe 2 (Figure 3). 
Ces deux groupes diffèrent essentiellement pour le rendement en grains, les nombres de grains par épi et par $\mathrm{m}^{2}$, la biomasse aérienne et le poids des épis, qui sont à l'avantage du groupe 1 (Figure 4). Les différences pour le nombre de plants levés, la capacité de tallage herbacée, la hauteur de la végétation, le col de l'épi et la surface de la feuille étendard ne semblent pas être déterminantes dans la réalisation d'une biomasse aérienne ni d'un rendement en grains élevés de l'échantillon des variétés évaluées.

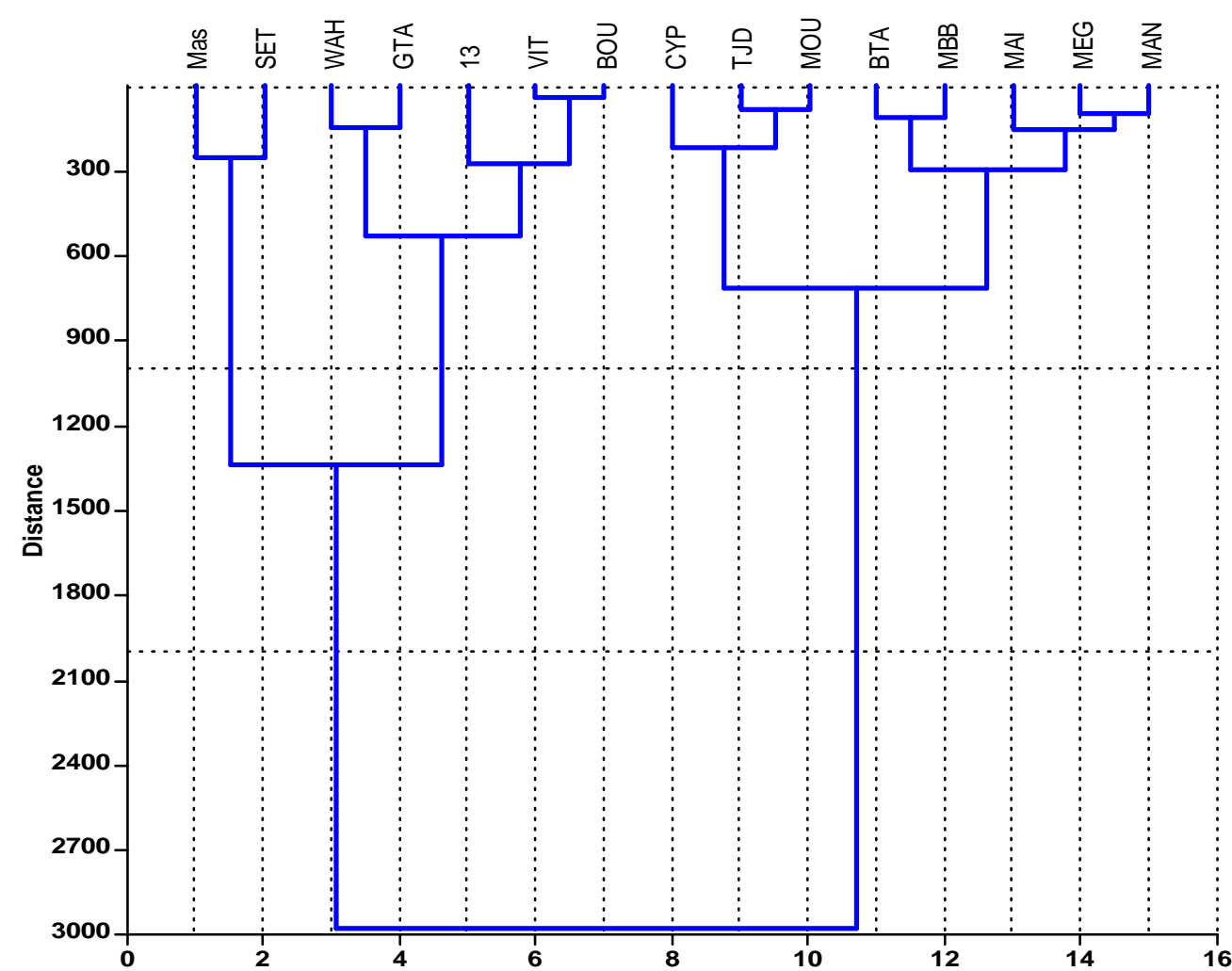

Fig.3. Groupage des génotypes évalués sur la base des moyennes de l'ensemble des dates et années étudiées. (Mas= Massara, Set13= Sétif2013,Cyp2= Cyprus2, MBB= Mohammed Ben Bachir, WAH= Waha, GTA= Gaviota durum, VIT $=$ Vitron, $\mathrm{MEG}=$ Megress, $\mathrm{SET}=$ Setifis, $\mathrm{BOU}=$ Bousselam, $\mathrm{MAN}=$ Mansoura, $\mathrm{MAI}=$ Massinissa, TJD= Tajdid, MOU= Moustakbel, BTA= Boutaleb).

En effet, l'analyse des corrélations de rangs entre les différentes variables mesurées montre que seuls la biomasse aérienne, le poids des épis et à un degré moindre le nombre de grains par épi sont les traits les plus liés avec le rendement en grains (Tableau 5). Ces résultats suggèrent que la sélection pour une biomasse, un poids des épis et un nombre de grains/épi élevés, conduirait à l'amélioration du rendement en grains, en moyenne des dates de semis et des années. 
Tableau 5. Coefficients de corrélations de rangs des différentes variables mesurées, moyennes génotypiques des dates et années (au-dessus de la diagonale probabilité et audessous de la diagonale, valeurs des coefficients de rangs de Sperman's).

\begin{tabular}{|l|l|l|l|l|l|l|l|l|l|l|l|l|l|}
\hline & PLT & Tll & PHT & SF & LC & DPV & BIO & PE & NE & NGE & NGM $^{2}$ & PMG & RDT \\
\hline PLT & & 0.283 & 0.390 & 0.567 & 0.919 & 0.805 & 0.879 & 0.428 & 0.098 & 0.093 & 0.524 & 0.869 & 0.321 \\
\hline Tll & 0.296 & & 0.647 & 0.408 & 0.357 & 0.520 & 0.226 & 0.084 & 0.002 & 0.929 & 0.108 & 0.889 & 0.063 \\
\hline PHT & -0.240 & -0.129 & & 0.328 & 0.294 & 0.354 & 0.129 & 0.203 & 0.904 & 0.293 & 0.273 & 0.731 & 0.198 \\
\hline SF & -0.161 & -0.231 & -0.271 & & 0.415 & 0.128 & 0.188 & 0.051 & 0.321 & 0.482 & 0.093 & 0.688 & 0.183 \\
\hline LC & 0.029 & -0.256 & 0.290 & -0.227 & & 0.597 & 0.558 & 0.286 & 0.879 & 0.687 & 0.985 & 0.037 & 0.202 \\
\hline DPV & -0.070 & -0.181 & 0.258 & -0.411 & 0.149 & & 0.240 & 0.815 & 0.965 & 0.404 & 0.516 & 0.592 & 0.480 \\
\hline BIO & 0.043 & 0.332 & 0.410 & -0.359 & -0.165 & 0.324 & & 0.008 & 0.058 & 0.620 & 0.034 & 0.498 & 0.017 \\
\hline PE & -0.221 & 0.461 & 0.349 & -0.513 & -0.295 & -0.066 & 0.657 & & 0.296 & 0.036 & 0.001 & 0.180 & 0.000 \\
\hline NE & 0.443 & 0.739 & 0.034 & -0.275 & 0.043 & -0.013 & 0.500 & 0.289 & & 0.226 & 0.243 & 0.638 & 0.324 \\
\hline NGE & -0.449 & -0.025 & 0.291 & -0.197 & 0.114 & -0.233 & 0.139 & 0.543 & -0.332 & & 0.003 & 0.481 & 0.014 \\
\hline NGM & -0.179 & 0.432 & 0.302 & -0.449 & 0.005 & -0.182 & 0.550 & 0.782 & 0.321 & 0.719 & & 0.879 & 0.000 \\
\hline PMG & -0.047 & 0.039 & 0.097 & 0.113 & -0.542 & -0.151 & 0.190 & 0.366 & 0.133 & -0.197 & -0.043 & & 0.224 \\
\hline RDT & -0.275 & 0.492 & 0.352 & -0.363 & -0.349 & -0.198 & 0.606 & 0.942 & 0.273 & 0.617 & 0.854 & 0.334 & \\
\hline
\end{tabular}

$\left(\mathrm{PLT}=\right.$ Plantes levées $/ \mathrm{m}^{2}, \mathrm{Tll}=$ talles herbacées $/ \mathrm{m}^{2}, \mathrm{PHT}=$ hauteur de la plante, $\mathrm{cm} ; \mathrm{SF}=$ surface de la feuille étendard, $\mathrm{cm}^{2}$; $\mathrm{LC}=$ longueur du col de l'épi, $\mathrm{cm}$; DPV=durée de la phase végétative, jours ; $\mathrm{BIO}=$ biomasse aéreinene mesurée à maturité, $\mathrm{g} / \mathrm{m}^{2} ; \mathrm{PE}=$ Poids de épis $/ \mathrm{m}^{2}, \mathrm{~g} / \mathrm{m}^{2} ; \mathrm{NE}=$ Nombre d'épis par $\mathrm{m}^{2}, \mathrm{NGM}^{2}=$ Nombre de grains par $\mathrm{m}^{2}, \mathrm{PMG}=$ Poids de 1000 rains, $\mathrm{g} ; \mathrm{RDT}=$ Rendement en grains, $\mathrm{g} / \mathrm{m}^{2}$ )

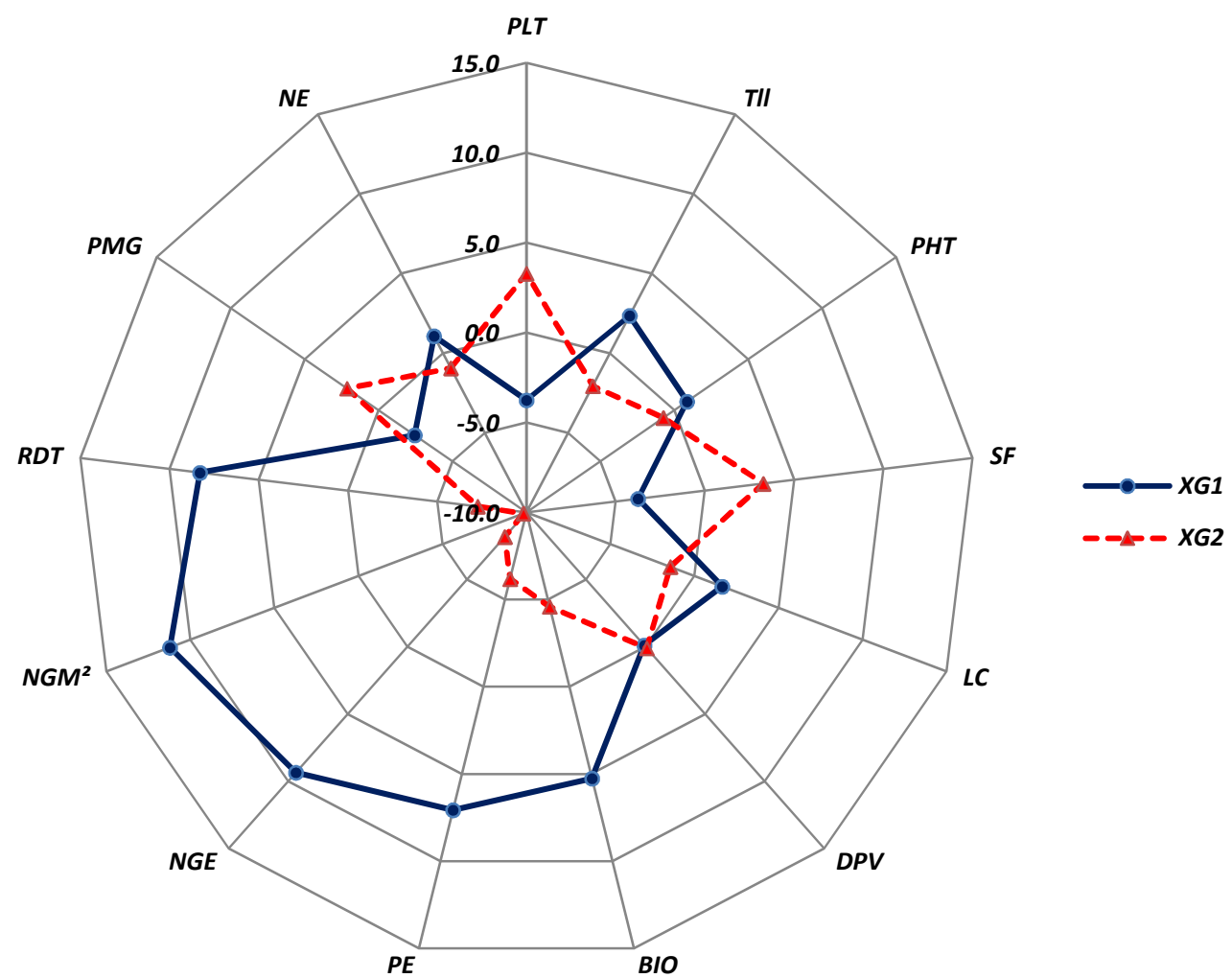

Fig.4. Différences relatives entre les deux groupes de génotypes évalués classés sur la base des moyennes des variables mesurées de l'ensemble des dates et années étudiées. (XG1= Massara, Waha, Setifis, Gaviota durum, Sétif2013, Vitron et Bousselam ; XG2= Cyprus2, MBB, Megress, Mansoura, Massinissa, Tajdid, Moustakbel et Boutaleb ; PLT = Plantes levées $/ \mathrm{m}^{2}, \mathrm{Tll}=$ talles herbacées $/ \mathrm{m}^{2}$, PHT = hauteur de la plante, $\mathrm{cm} ; \mathrm{SF}=$ surface de la feuille étendard, $\mathrm{cm}^{2} ; \mathrm{LC}=$ longueur du col de l'épi, $\mathrm{cm} ; \mathrm{DPV}=$ durée de la phase végétative, jours ; $\mathrm{BIO}=$ biomasse aéreinene mesurée à maturité, $\mathrm{g} / \mathrm{m}^{2} ; \mathrm{PE}=$ Poids de épis $/ \mathrm{m}^{2}, \mathrm{~g} / \mathrm{m}^{2} ; \mathrm{NE}=$ Nombre d'épis par $\mathrm{m}^{2}, \mathrm{NGM}^{2}=$ Nombre de grains par $\mathrm{m}^{2}, \mathrm{PMG}=$ Poids de 1000 rains, $\mathrm{g} ; \mathrm{RDT}=$ Rendement en grains, $\mathrm{g} / \mathrm{m}^{2}$ ). 


\subsection{Effet date de semis}

En moyenne des 15 génotypes et des trois années d'études, le semis précoce présente un avantage significatif comparativement au semis tardif pour presque l'ensemble des variables mesurées (Tableau 6). En effet, le semis précoce se distingue par une épiaison plus précoce de 6.7 jours, en moyenne. Ceci présente l'avantage de soustraire une partie du cycle de la plante aux effets pénalisants des stress abiotiques de fin de cycle de la culture, tels que le déficit hydrique et les hautes températures.

Tableau 6. Valeurs moyennes des variables mesurées de l'effet date de semis (moyenne des 15 génotypes et trois années).

\begin{tabular}{|l|l|l|l|l|l|l|l|l|l|l|l|l|l|}
\hline DATE & PLT & TII & PHT & SF & LC & DPV & BIO & PE & NE & NGE & NGM $^{2}$ & PMG & RDT \\
\hline D1 & 227.4 & 745.1 & 65.7 & 17.3 & 21.0 & 120.3 & 742.2 & 407.5 & 288.3 & 21.3 & 6565.7 & 40.7 & 277.4 \\
\hline D2 & 237.5 & 697.9 & 51.6 & 13.3 & 20.2 & 126.7 & 622.2 & 347.3 & 318.8 & 16.7 & 5984.9 & 37.7 & 230.0 \\
\hline Ppds5\% & 3.5 & 9.5 & 0.4 & 0.2 & 0.2 & 0.0 & 11.9 & 4.3 & 4.8 & 0.4 & 98.5 & 0.4 & 3.4 \\
\hline
\end{tabular}

$\left(\mathrm{PLT}=\right.$ Plantes levées $/ \mathrm{m}^{2}, \mathrm{Tll}=$ talles herbacées $/ \mathrm{m}^{2}, \mathrm{PHT}=$ hauteur de la plante, $\mathrm{cm} ; \mathrm{SF}=$ surface de la feuille étendard, $\mathrm{cm}^{2}$; LC= longueur du col de l'épi, $\mathrm{cm}$; DPV=durée de la phase végétative, jours ; $\mathrm{BIO}=$ biomasse aéreinene mesurée à maturité, $\mathrm{g} / \mathrm{m}^{2} ; \mathrm{PE}=$ Poids de épis $/ \mathrm{m}^{2}, \mathrm{~g} / \mathrm{m}^{2} ; \mathrm{NE}=$ Nombre d'épis par $\mathrm{m}^{2}, \mathrm{NGM}^{2}=\mathrm{Nombre}$ de grains par $\mathrm{m}^{2}, \mathrm{PMG}=$ Poids de 1000 rains, g ; RT = Rendement en grains, g/m² ).

Le semis précoce présente aussi un avantage, de plus de $10 \mathrm{~cm}$, pour la hauteur de la végétation, et de plus de $4 \mathrm{~cm}$ pour la surface de la feuille étendard. Les écarts moyens, entre dates de semis, du tallage herbacé $\left(+47.2\right.$ talles $\left./ \mathrm{m}^{2}\right)$, de la biomasse aérienne $\left(+120.0 \mathrm{~g} / \mathrm{m}^{2}\right)$, du poids des épis $\left(+60.2 \mathrm{~g} / \mathrm{m}^{2}\right)$, du nombre de grains par épi (+4.7 grains par épi), du nombre de grains par $\mathrm{m}^{2}\left(+580.8\right.$ grains $\left./ \mathrm{m}^{2}\right)$, du poids de 1000 grains $(+3.0 \mathrm{~g})$ et du rendement en grains $\left(+47.4 \mathrm{~g} / \mathrm{m}^{2}\right)$ sont à l'avantage du semis précoce, en moyenne des trois années et des 15 génotypes.

L'effet date de semis ne semble pas avoir un effet significatif sur la longueur du col, le nombre de plants levés et le nombre d'épis par $\mathrm{m}^{2}$ (Tableau 6). Par contre la hauteur de la paille est réduite de $21.1 \%$, la surface de la feuille étendard de $23.0 \%$, la biomasse aérienne de $16.2 \%$, le poids des épis de $14.8 \%$, le nombre de grains par épi de $21.9 \%$ et le rendement en grains de $17.1 \%$ (Figure 5).

Le nombre d'épis augmente de $10.6 \%$. Il est probable dans ce cas, que les pluies tardives, notamment celles observées au cours des campagnes 2014 et 2015, ont favorisé la montée en épis des talles tardives qui ne contribuent pas réellement au rendement en grains, mais constitue une gêne pour la récolte. Le nombre de plants s'améliore de $4.5 \%$ en semis tardif, ceci s'explique par le fait que le sol est plus humide lors du semis tardif, suite aux avènements pluvieux qui sont plus nombreux qu'en semis précoce. 


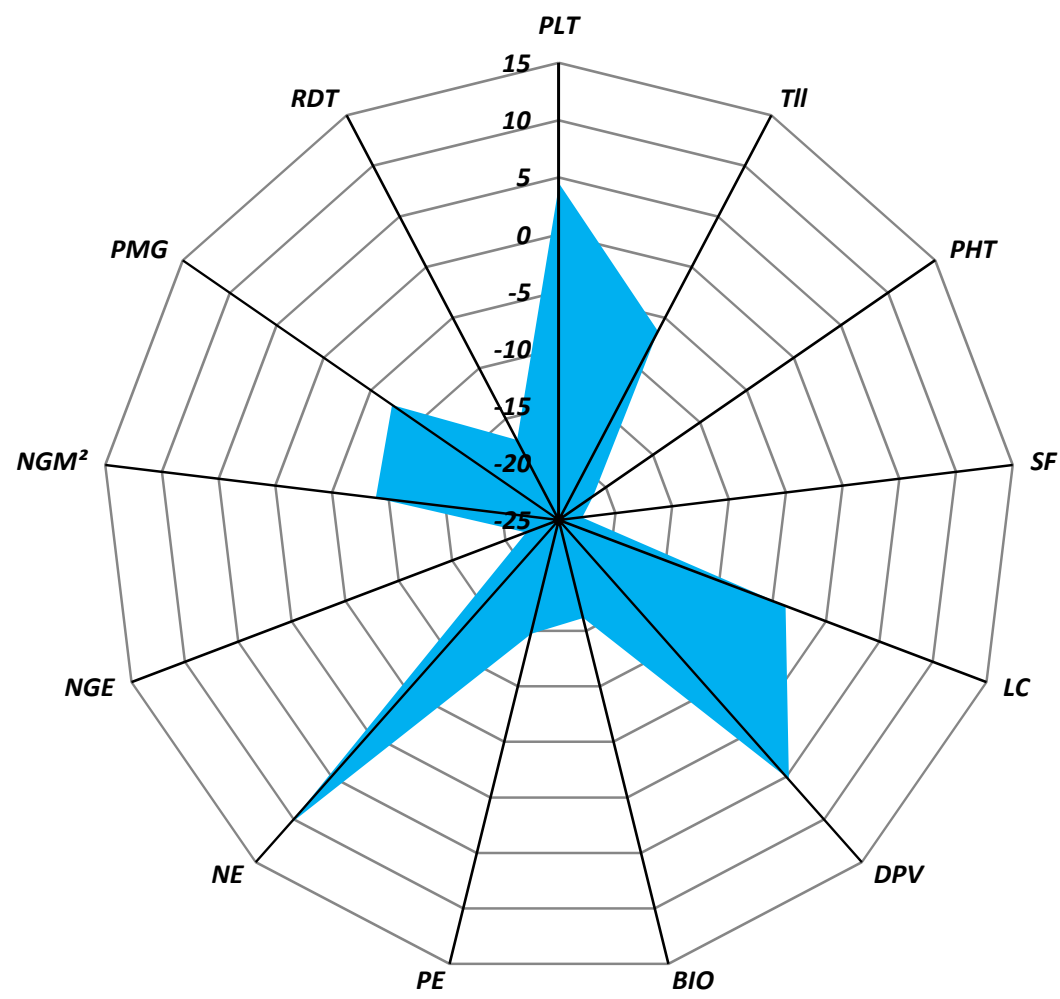

Fig.9. Réduction des valeurs moyennes des variables mesurées de la date tardive comparativement à la date précoce. (moyennes de trois années et des 15 génotypes, PLT= Plantes levées $/ \mathrm{m}^{2}, \mathrm{Tll}=$ talles herbacées $/ \mathrm{m}^{2}, \mathrm{PHT}=$ hauteur de la plante, $\mathrm{cm} ; \mathrm{SF}=$ surface de la feuille étendard, $\mathrm{cm}^{2}$; $\mathrm{LC}=$ longueur du col de l'épi, $\mathrm{cm}$; DPV=durée de la phase végétative, jours ; $\mathrm{BIO=biomasse} \mathrm{aéreinene} \mathrm{mesurée}$ à maturité, $\mathrm{g} / \mathrm{m}^{2} ; \mathrm{PE}=$ Poids de épis $/ \mathrm{m}^{2}, \mathrm{~g} / \mathrm{m}^{2} ; \mathrm{NE}=$ Nombre d'épis par $\mathrm{m}^{2}, \mathrm{NGM}^{2}=$ Nombre de grains $\mathrm{par} \mathrm{m}^{2}$, $\mathrm{PMG}=$ Poids de 1000 rains, $\mathrm{g} ; \mathrm{RDT}=$ Rendement en grains, $\mathrm{g} / \mathrm{m}^{2}$ )

Ces résultats suggèrent que le semis précoce favorise une meilleure expression des caractères qui sont, le plus souvent, les déterminants du rendement en grains, comparativement au semis tardif. Par conséquence la variation des performances du génotype serait moins importante en semis précoces qu'en semis tardifs, vu que les conditions de croissance sont, souvent, plus favorables en semis précoces qu'en semis tardifs.

\section{CONCLUSION}

L'étude de la variabilité phénotypique à travers l'effet moyens années, génotypes et date de semis fait ressortir :

Les campagnes 2014 et 2015 ont enregistré une baisse de l'ordre de 20 à $80 \%$, de l'expression des caractères étudiés en comparaison à la campagne 2013. Une réduction qui touche aussi bien l'ensemble des caractères constituants l'architecture de la plante que le rendement. Il est alors important d'aller chercher la performance entre les variétés puisqu'il est difficile de la prédire par années. 
L'effet moyen génotype montre une différence significative entre les génotypes pour l'ensemble des caractères, une différence qui fait probablement suite à la présence de l'interaction des génotypes avec les dates et les années. Ainsi l'analyse de l'amplitude des moyennes des caractères étudiés, et qui se situe entre 10 et $56 \%$, offre des possibilités de sélection au sein de l'échantillon de variétés étudiées. Le Groupage des génotypes évalués sur la base des moyennes de l'ensemble des dates et années étudiées et en plus de l'analyse des corrélations de rangs indiquent que la sélection pour une biomasse, un poids des épis et un nombre de grains par épi et par $\mathrm{m}^{2}$ élevés, conduirait éventuellement à l'amélioration du rendement en grains, en moyenne des dates de semis et des années. C'est le cas de figure de la variété Setifis qui présente les valeurs, les plus élevées, pour le poids des épis, les nombres de grains par épi et par $\mathrm{m}^{2}$ et le rendement en grains.

L'effet date de semis indique que le semis précoce présente un avantage significatif comparativement au semis tardif pour presque l'ensemble des variables mesurées, en moyenne des 15 génotypes et des trois années d'études. Ainsi l'épiaison précoce, la hauteur de la végétation, la surface de la feuille étendard, le tallage herbacé, la biomasse aérienne, le poids des épis, le nombre de grains par épi, le nombre de grains par $\mathrm{m}^{2}$, le poids de 1000 grains et le rendement en grains sont à l'avantage du semis précoce. Le semis précoce favorise une meilleure expression des caractères qui sont, le plus souvent, les déterminants du rendement en grains, comparativement au semis tardif.

\section{ACKNOWLEDGEMENTS}

This research was undertaken as a part of the project CNEPRU $\mathrm{N}^{\circ}$ F01220140023 titled 'Variability, genetic determinism and selection of cereals under semi- arid conditions of the Algerian eastern high plains', financed by the Algerian Ministry of Higher Education and Scientific Research. Special thanks are extended to the personnel of the ITGC-ARS and INRAA-RUS, particularly to Mrs Djamel Sersoub, Houcine Zerargui, Abderrahmane Hannachi and Adel Bachir for providing seeds, helping in the management of the trials and data collection.

\section{REFERENCES}

[1] Zahid A. Mécanismes cellulaires et moléculaires régissant le métabolisme des semences de céréales : Rôle du réseau rédoxines- Système antioxydant dans la prédiction de la qualité germinative, 2010, Thèse de Doctorat de l'université de Toulouse, Pp 60. 
[2] Chennafi H, Aïdaoui A, Bouzerzour H. Saci A. Yield response of durum wheat (Triticum durum Desf.) cultivar Waha to deficit irrigation under semi-arid growth conditions. Asian J. Plant Sci, 2006, 5, 854-860.

[3] Bahlouli F, Bouzerzour H, Benmahammed A, Hassous K. L. Selection of high yielding and risk efficient durum wheat (Triticum durum Desf.) cultivars under semi-arid conditions. Pak. J. Agron, 2005, 4, 360-365.

[4] Laala Z, Oulmi A, Saraoui T, Haddad L, Nouar H, Benmahammed A, Bouzerzour H. Effet de la sélection de la biomasse et des épis sur le rendement du blé dur (Triticum durum Desf.) sous conditions semi-arides. Annales de la Faculté des Sciences et Sciences de l'Ingénieur, 2009, 1, (4), 56- 69.

[5] Bouzerzour H, Dekhili M. Heritabilities, gains from selection and genetic correlations for grain yield of barley grown in two contrasting environments. Field Crops Res, 1995, 41, 173178.

[6] Nouar H, Bouzerzour H, Haddad L, Menad A, Hazmoune T, Zerargui H. Genotype x Environment interaction assessment in durum wheat (Triticum durumDesf.) using AMMI and GGE Models. Advances in Environmental Biology, 2012, 6, 3007-3015.

[7] Benmahammed A, Nouar H, Haddad L, Laala Z, Oulmi A, Bouzerzour H. Analyse de la stabilité des performances de rendement du blé dur (Triticum durum Desf.) sous conditions semi-arides. Biotechnol. Agron. Soc. Environ, 2010, 14, 177-186.

[8] Munns R, James J. A, Xu B, Athman A, Conn S. J, Jordans C, Byrt C. S, Hare R. A, Tyerman S. D, Tester M, Plett D, Gilliham M. Wheat grain yield on saline soils is improved by an ancestral Na+transporter gene. Nature Biotechnology, 2012, 30, 360 - 364.

[9] Annicchiarico P, Bellah F, Chiari T. Defining sub regions and estimating benefits for a specific adaptation strategy by breeding programs: a case study. Crop Sci, 2005, 45, 17411749 .

[10] Debaeke P, Casadebaig P, Haquin B, Mestries E, Palleau J.P, Salvi F. Simulation de la réponse variétale du tournesol à l'environnement à l'aide du modèle SUNFLO. OCL Oléagineux, Corps Gras, Lipides , 2010, 17, 143-151.

[11] Kadi Z, Adjel F, Bouzerzour H. Analysis of the genotype x environment interaction of barley grain yield (Hordeum Vulgare L.) under semi-arid conditions. Advances in environmental biology, 2010, 4, 30-40.

[12] Adjabi A, Bouzerzour H, Benmahammed A, 2014. Stability Analysis of Durum Wheat (Triticum durum Desf.) Grain Yield. Journal of Agronomy, 2014, 13, 131-139. 
[13] Kribaa M, Hallaire V, Curmi P, Lahmar R. Effects of various cultivation methods on the structure and hydraulic properties of soil in semi-arid climate. Soil Tillage Res, 2001, 60, 4353.

[14] CropStat. Logiciel de statistique, 2009, version 7.2.3., IRRI, Manilla.

\section{How to cite this article:}

Haddad L, Bouzerzour H, Benmahammed A, Zerargui H, Hannachi A, Bachir M, Salmi M, Oulmi A, Nouar, H, Laala Z. Analysis of the phenotypic variability of some varieties of durum wheat (triticum durum desf) to improve the efficiency of performance under the constraining conditions of semi-arid environments. J. Fundam. Appl. Sci., 2016, 8(3), 10211036. 